\title{
Detection of friction-modulated textures is limited by vibrotactile sensitivity
}

\author{
Corentin Bernard, Sølvi Ystad, Jocelyn Monnoyer and Michaël Wiertlewski
}

\begin{abstract}
Modulation of the frictional force of a fingertip sliding over a surface-haptic device can produce compelling sensations of texture and relief. The virtual sensation is particularly apparent and feel as fixed in space if the stimulus is rigorously correlated with the displacement of the finger. While frictional textures tactually resemble their real counterparts, some exploratory conditions under which the sharpness of the texture declines exist. We postulate that this decline in sharpness is caused by the perceptual limitation of the attempt to interpret the variation in friction as an out-of-plane sinusoidal topography. To investigate these questions, we measured the detection thresholds of sinusoidal friction-modulated gratings for a wide range of spatial periods explored at two different speeds. We compared the results with the detection thresholds, reported in the literature, of real gratings and vibrotactile stimuli. We found that the detection of spatial friction-modulated textures does not follow the same trend as that of real textures but is more similar to the vibrotactile rendering, which is strongly influenced by the exploratory speed. This study provides a better understanding of the perception of friction-modulated textures and provides insight into how to design impactful stimuli on surface-haptic devices.
\end{abstract}

Index Terms - Haptics, psychophysics, friction modulation, tactile perception, detection thresholds, finger velocity, touchscreen.

\section{INTRODUCTION}

Sher URFACE-haptics is a promising way to enhance the human-machine interaction by providing localized sensations on a touchscreen. These technologies lend themselves to a large number of applications such as consumer electronics and the automotive industry, with the promise of decreasing visual distraction. Owing to the fine control over the frictional force on the skin, these devices also provide an opportunity for furthering the understanding of human tactile perception.

Changes in friction between the user finger and the glass plate can provide the sensation of touching shapes that protrude from the plate or even fine textures. The mechanism behind the perceptual integration of frictionmodulated spatial patterns into believable tactile textures is still an open question. Friction changes affect the entire contact surface and can vary only over time; yet, the perceptual experience is convincingly similar to touching a physical relief, the features of which are distributed in space.

For the relief to be perceived in a coherent way, it must be precisely localized. Therefore, friction is usually modulated as a function of the finger position, i.e., each finger position corresponds to one friction level. The illusion breaks down if the presentation of the stimulus lags behind the user's motion [1].

Since the frictional force can be modulated only over the whole fingertip, creating synthetic curvatures smaller than the area of contact should be impossible. However, friction variations below this limit produce vibratory oscillations

- C. Bernard and S. Ystad are with Aix-Marseille Univ, CNRS, PRISM, Marseille, France. E-mail : bernard@prism.cnrs.fr

- C. Bernard and J. Monnoyer are with the Groupe PSA, Paris, France. and Aix-Marseille Univ, CNRS, ISM, Marseille, France.

- M. Wiertlewski is with TU Delft, Delft, The Netherlands.

This work was conducted in the framework of the Openlab PSA-AMU "Automotive Motion Lab". MW acknowledges the support of the Agence Nationale de la Recherche project IOTA ANR-16-CE33-0002 that give the illusion of touching fine textures in a fashion similar to that of vibrotactile stimuli [2].

For virtual as well as real textures, the frequency of the vibrations produced by the skin-surface contact that propagate at the surface of the skin shifts according to the exploration velocity. Vibrations are perceptually integrated with exploratory motion as a unique and invariant percept. These vibrations are believed to be one of the main factors that mediate texture perception [3], [4], [5]. Human detection of vibrotactile stimuli has been extensively studied [6], [7].

The sensitivity to vibrations follows a U-curve from $10 \mathrm{~Hz}$ up to $800 \mathrm{~Hz}$, with an optimal sensitivity at $250 \mathrm{~Hz}$. This sensitivity curve was found when subjects were static and the apparatus presented a fixed stimulus. In contrast, when touching real or surface-haptic textures, the observer has to conduct an active exploration to acquire relevant informations. In this context the skin is in relative motion with the display, causing a fundamentally different mechanical interaction.

Natural textures, in contrast, have a much richer interaction than fixed vibrotactile stimuli. Louw et al [8] showed that the detection thresholds for real Gaussian bumps follow a linear trend with respect to the spatial period of the features. The results were subsequently extended to sinusoidal gratings [9]. The authors found that the preponderant factor in the determination of the detection threshold was the slope of the relief. The minimal perceptible gradient is $1.3 \mu \mathrm{m} / \mathrm{mm}$ over a large range of spatial scales from a few hundred microns to several centimetres. In these experiments, subjects were free to explore the textures without any speed restriction. It is thus not possible to deduce the spectral content of the vibrations that propagated in the finger from this study.

These results lead us to question which mechanism is involved in the perception of friction-modulated gratings: Are 
spatially defined frictional textures perceptually integrated as a spatial topography or as time-varying vibrotactile signals?

To investigate this question, the present paper studies the detection thresholds of spatially defined sinusoidal gratings of various spatial periods under two exploration velocity conditions using ultrasonic friction modulation technology. For the same spatial period $\lambda$, the induced vibration frequency $f$ is affected by the scanning speed $v$, following the relation $f=v / \lambda$. The faster the exploration speed is, the higher the vibration frequency. Our assumption is that if these textures are spatially integrated, the detection threshold of a grating at a given spatial period $\lambda$ will not be affected by the scanning speed, as presented in Fig. 1.a (left). Consequently, a difference occurs between the two finger velocities if we compare the detection thresholds of the induced vibration frequencies $f=v / \lambda$, as presented in Fig. 1.a (right). In the opposite case, if the textures are temporally integrated, the detection thresholds of the stimuli should be guided by the vibration frequencies $f$ produced during the exploration, as presented in Fig. 1.b (right). In this case, the thresholds are invariant with finger velocity in the frequency domain, which implies that the detection threshold of a grating at a given spatial period $\lambda$ will vary with the finger velocity $v$, as presented in Fig. 1.b (left).

To measure the friction variations induced by the stimuli around perception thresholds, we designed a custom force sensor sensitive to stimuli four times smaller than the detection threshold of a human observer. In addition, we instrumented physical quantities that are relevant to texture perception, such as the amplitude of the plate vibration, the vibration of the skin and the subject's perception. The setup assesses all the physical quantities involved in the perception of friction-modulated texture.

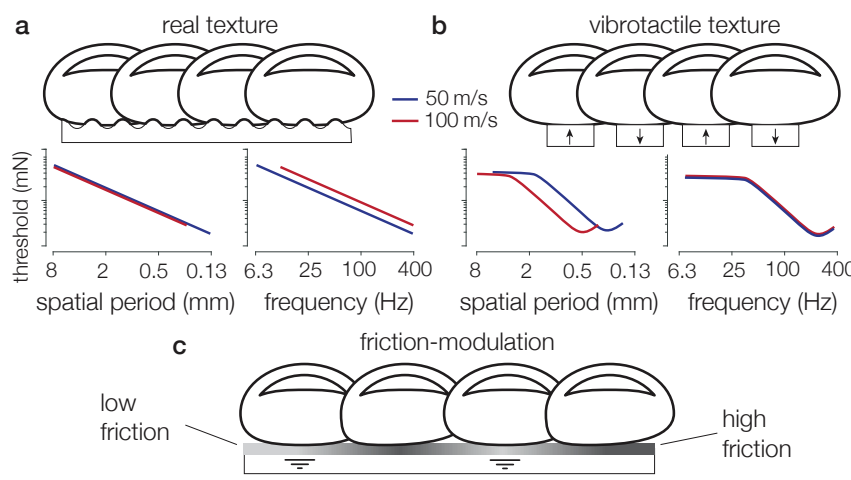

Fig. 1. a. Detection threshold curve with a spatially based perception model in the spatial and frequency domains. Data extracted from [8], [9]. b. Detection threshold curve with a frequency based perception model in the spatial and frequency domains. Data extracted from [6] $50 \mathrm{~mm} / \mathrm{s}$ and $100 \mathrm{~mm} / \mathrm{s}$ velocities are shown in blue and red respectively. c. Interaction with a virtual texture on a surface-haptic device.

\section{BACKGROUND}

Our experiment was designed on the basis of many previous investigations on human tactile sensory systems, especially those on the perception of friction modulation.

\subsection{Perception of physical textures}

\subsubsection{Mechanoreceptors and afferents involved}

The tactile sensory system is believed to be mediated by four types of mechanoreceptors in the skin [10]. Three of which appear to be relevant to texture perception. Each of these three types of mechanoreceptors contributes to the encoding of a part of the frequency spectrum of the vibration generated during the exploration of textured surfaces. Merkel corpuscles, responsible for slowly adapting type I afferents (SA I), are sensitive to skin deformations in a limited low-frequency bandwidth under $5 \mathrm{~Hz}$. Meissner corpuscles, associated with Fast-adapting type I afferents (FA I), respond to frequencies between 5 and $40 \mathrm{~Hz}$. Pacinian corpuscles, responsible for fast-adapting type II afferents (FA II), are particularly sensitive to vibrations from 40 to $400 \mathrm{~Hz}$, with an optimal sensitivity of approximately $250 \mathrm{~Hz}$. The involvement of every afferent in encoding the sensation of texture varies with the size of the asperities and the speed of exploration [11].

\subsubsection{Influence of the exploration speed}

For a long time, texture perception was believed to follow the so-called duplex theory [12], which suggests that coarse textures are determined by spatial cues and that fine textures are determined by temporal cues [13]. This theory is supported by the fact that movement plays a crucial role in texture discrimination [14]. Indeed, roughness estimation of real gratings is affected by their spatial period [15], but not by the finger velocity [16]. However, recent studies have shown that spatial and temporal codes cannot be easily segregated [11]. Even if the vibration frequency generated by texture exploration depends on the finger velocity, a relative invariance to speed remains in its discrimination [5], and their spatial spectra are similar [17]. Illusions of exploring textures without directly using the finger can be rendered by enhancing a pen with vibrations recorded on real textures [18], [19], but the frequency content of these vibrations must be adapted to the exploration velocity [20]. When proprioceptive cues are absent, the perceived scanning speed is affected by the frequency content [21] [22]. These studies reveal the intricate link between textures, vibrations and movement.

\subsection{Virtual textures}

\subsubsection{Surface haptics technologies}

There are mainly three kinds of haptic surface technology. The most common is based on vibrotactile feedback and uses mechanical vibrations of the screen in a frequency range adapted to human sensitivity to create illusions of buttons and textures [2], [23], [24], [25] that can even be localized [26]. However, this technology cannot render lowfrequency variations. Another technology uses electroadhesion to modulate friction by increasing electrostatic forces to attract the finger to the plate [27]. Compared to ultrasonic friction modulation, electroadhesion offers a wider frequency bandwidth of modulation but a narrower range of friction variations.

The technology used in the present study is based on ultrasonic friction modulation. A glass plate vibrates at 
a resonant high-frequency mode (above $30 \mathrm{kHz}$ ). These vibrations are not perceptible, but they slightly lift the finger off the plate. The near-field acoustic levitation of the finger reduces the friction between the finger and the screen [28]. This friction reduction can be modulated to create illusions of textures or shapes on a glass plate [29], [30].

\subsubsection{Perception of friction modulation}

Many studies have been performed to better understand how humans perceive ultrasonic friction modulation. Some focused on transient changes in friction that render the illusion of passing a finger over a frontier [31]. A higher finger velocity was found to increase the intensity of the perception [32]. Interestingly, it has been shown that transient changes in friction could also be perceived with static touch [33] to recreate the illusion of pressing a button. Other studies have shown that the perception of two superimposed friction modulated sinusoidal gratings presents masking effects [34], [35]. Roughness estimation of square gratings as a function of their spatial period appears to follow opposite trends between real textures and their synthetic counterpart [36].

Experiments on the detection thresholds of friction modulation have shown a correlation with the friction level [37], with a better sensitivity for low finger velocities. Psychophysical studies have also been performed to understand how modulated friction is perceived with electroadhesion [38]. It has been found that harmonic frequencies affect the detection thresholds of a periodic pattern [39].

\section{MATERIALS AND METHOdS}

\subsection{Design motivation}

\subsubsection{Experimental setup}

The experimental setup was designed with the objective of providing a highly consistent signal by ensuring a spatial and temporal resolution of the stimulus orders-ofmagnitude higher than the perceptual limits. In addition, the quantification of the output signal was sufficiently fine to avoid any artefacts. The spatial stimuli were created by designing a friction map; a function of the desired friction level according to the finger position. Every $200 \mu \mathrm{s}$, the controller polled the finger position and sent the actuation command.

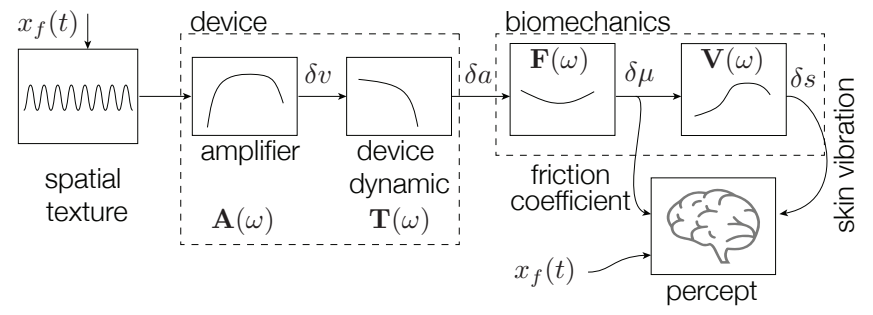

Fig. 2. The signal is generated from a lookup table indexed as a function of the user's position $x_{f}(t)$. The signal is then amplified and the voltage modulation $\delta v$ is sent to the piezoelectric actuators. Changes in the ultrasonic vibrations of the plate $\delta a$ result in changes in the frictional force $\delta \mu$, which creates skin vibrations, captured by an accelerometer on the skin surface on the first phalanx of the finger $\delta v$.

\subsubsection{Psychophysics and perceptual transfer functions}

Fig. 2 summarizes the physical quantities that are related to the perception of frictional texture, from the actuation to the perception. The algorithm used in the threshold experiment presents a stimulus defined as an amplitude modulation command. This command is converted into a voltage modulation that mixes with the carrier frequency and is provided to the actuators on the glass plate. The effective vibration modulation of the plate, which depends on its dynamics, induces squeeze-film levitation which in turn modulates the frictional forces between the finger and the plate. Friction changes at the contact between the skin and the glass plate produce mechanical vibrations that propagate into the whole finger. Since the precise relevance of friction force and vibration of the skin in the perceptual experience of tactile texture is unclear, we measured both variables during the psychophysical experiment. Fig. 3 shows the experimental setup, and the technical details are described in the following section.

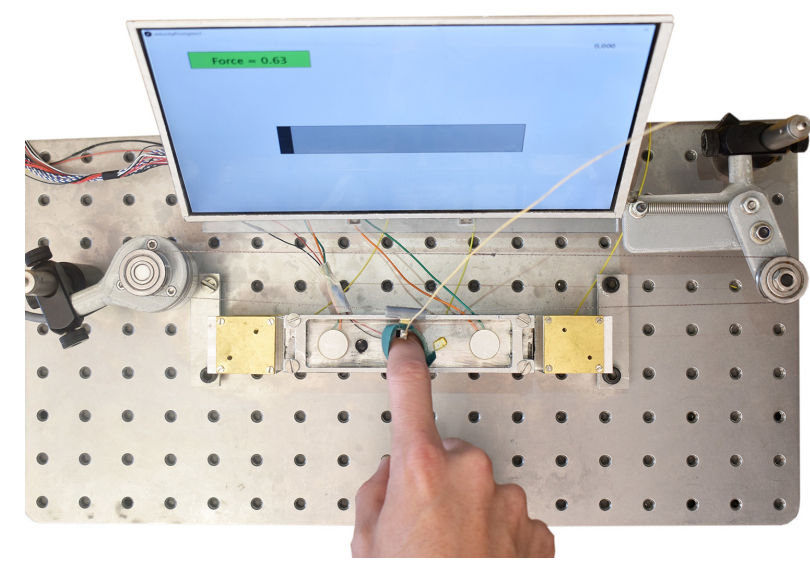

Fig. 3. Experimental setup. The subject touches the actuated glass plate on top of the force sensor. The finger is linked to a pulley-encoder system for position measurement and an accelerometer is placed on the first phalanx. The screen shows a cursor imposing the velocity and provides feedback on the normal force.

\subsection{Texture production on surface-haptic devices}

The finger position is tracked with a small ring attached to the participant's finger. The ring is connected to a pulleyencoder system that measures unidirectional finger displacements along the length of the glass plate. The precision of this system is approximately $0.01 \mathrm{~mm}$ and can be captured at $4 \mathrm{kHz}$ without any significant latency. A microcontroller (Teensy 3.5) reads the encoder and outputs a modulating signal according to a friction map encoded in memory on a fixed real-time timer. The carrier signal, a $35 \mathrm{kHz}$ sine wave, is created by a function generator (BK Precision 4052) and amplitude modulated by the analog signal coming from the microcontroller. The resulting signal is then amplified 20-fold (WMA-100, Falco Systems) to drive two piezoelectric actuators glued on a $105 \times 22 \times 3.3 \mathrm{~mm}$ glass plate. Modulation of the amplitude of vibration of the glass plate induces friction variations during exploration. 


\subsection{High-precision force sensor}

To measure the interaction force acting on the participants' finger, we designed a custom sensor able to measure forces with a better sensitivity than that of the human sensory system, over its entire sensitive frequency range [6]. The force sensor is based on a rigid elastic structure in which nanometre-scale deformation is measured via a Fabry-Perot interferometer. The sensor is optimized to cover a frequency bandwidth that spans continuous forces to stimulation up to $800 \mathrm{~Hz}$, and is able to resolve forces with amplitudes lower than $1 \mathrm{mN}$.

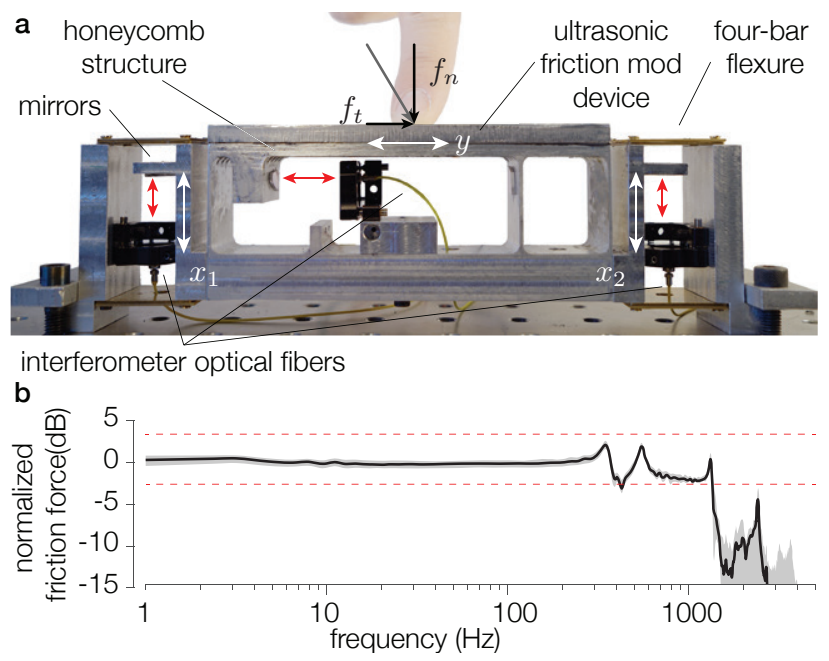

Fig. 4. a Picture of the tribometer. The glass plate is mounted on a honeycomb structure that provides light and stiff support. The test sample is suspended by a set of three four-bar flexures whose deflection is measured with an interferometer via fibre optics. Red arrows show the laser path between the fibre optics and the mirrors. b Frequency response of the sensor. The median of ten normalized lateral displacement amplitudes is printed in black and its first and third quartiles are shaded in grey. Red dashed lines show the $\pm 3 \mathrm{~dB}$ range.

\subsubsection{Mechanical structure}

The structure of the force sensor is presented in Fig. 4.a. The actuated glass plate is fixed to the top of an aluminium support with a lightweight yet stiff honeycomb structure. The aluminium support is suspended by three flexures that allow for minute lateral displacements but are virtually infinitely stiff in the other directions. This structure guides the deformation along the sensing axis of the transducer. The lateral force sensor is then suspended above the ground with two brass four-bar flexure linkages on each side. Similar to the lateral flexures, these horizontal brass flexures allow for vertical movements while limiting motion in the other degrees of freedom.

\subsubsection{Deformation measurement and calibration}

The deformation of the structure is measured by a 3-axis Fabry-Perot interferometer (IDS 3010, Attocube). The laser is guided by an optical fibre and focused by a lens (D4/F1, Attocube), which is mounted on the base of the structure. The laser is then reflected to the lens by a mirror, mounted on the flexible part of the structure. Micrometric adjustment screws enable alignment of the laser path. The picometersized displacements of the three axes ( 2 normal $x_{1}$ and $x_{2}$ and one tangential to the fingertip exploration $y$ ) are then converted into an analog output refreshed at $10 \mathrm{MHz}$. The calibration is performed by first applying a known force to the upper part of the sensor with a mass and then by using the same weight with a string and pulley system to apply a tangential load.

\subsubsection{Performance}

The frequency response of the structure to a tangential impact on the top plate is shown in Fig. 4.b. The frequency response shows a main cut-off frequency of approximately $1300 \mathrm{~Hz}$, with some normal modes between $300 \mathrm{~Hz}$ and $600 \mathrm{~Hz}$. The measured noise floor of the presented structure and interferometer system is $0.4 \mathrm{mN}$.

\subsection{Additional sensors}

In addition to the 2-axis force sensor, an accelerometer (Model 2250A / AM1-10, Meggitt) with a flat response over a frequency bandwidth spanning from 2 to $15000 \mathrm{~Hz}$, is attached to the plastic ring in contact with the skin of the first phalanx to measure the propagation of vibrations into the surface of the skin of the index finger. Indeed the vibrations from the tactile interaction propagate in the whole hand [40]. The finger vibrations, contact forces and finger positions are recorded with an acquisition card (USB $X$ Series Multifunction DAQ, National Instruments) at a $10 \mathrm{kHz}$ sampling rate preceded by an antialiasing filter. To measure the vibration of the plate, a third piezoelectric ceramic acting as a sensor is glued to the glass plate. The input voltage applied to the piezoelectric actuator and the output voltage from the piezoelectric sensor are recorded by a dedicated acquisition card (NI USB-6211, National Instruments) at a $100 \mathrm{kHz}$ sampling rate, to provide enough resolution for demodulation of the ultrasonic wave. The output signal is calibrated with an interferometer (IDS 3010, Attocube) to obtain the glass plate vibration in micrometres. Before and after each session, the moisture on the subjects' index finger is assessed with a dedicated device (gpskin Barrier pro). This measurement does not provide any insight into the results and will therefore not be discussed.

\subsection{Psychophysical experiment}

\subsubsection{Participants}

17 volunteers, 5 females and 12 males, 15 right-handed and 2 left-handed, ranging from 22 to 42 years old (mean 28.2) participated in the study. They were naive to the aims of the study and none of them reported having any skin concerns. The study was approved by the Ethical Committee of AixMarseille University. The participants gave their informed consent before the experiment. They were paid for their participation. They washed and dried their hands and the glass plate was cleaned with an alcoholic solution before the experiment. The results of two subjects were discarded because of technical issues.

\subsubsection{Stimuli}

We investigated the subjects' detection thresholds for friction-modulated sine waves spatially encoded by the position of the finger. The experiment was divided into two 
parts corresponding to the two finger velocity conditions (50 mm/s and $100 \mathrm{~mm} / \mathrm{s})$. The presentation order of the velocity condition was alternated between subjects. For the $50 \mathrm{~mm} / \mathrm{s}$ velocity, haptic stimuli were rendered for 7 spatial periods: $0.125,0.25,0.5,1,2,4$ and $8 \mathrm{~mm}$. For the $100 \mathrm{~mm} / \mathrm{s}$ velocity, haptic stimuli were rendered for 6 spatial periods: $0.25,0.5,1,2,4$ and $8 \mathrm{~mm}$. According to the 2 finger velocities, the vibrations transmitted to the finger varied from $6.25 \mathrm{~Hz}$ to $400 \mathrm{~Hz}$. The $0.125 \mathrm{~mm}$ condition was not presented for the high velocity since it would produce a fundamental frequency of $800 \mathrm{~Hz}$, which could not be rendered by the glass plate. The spatial period sessions were presented in random order. The experiment lasted for approximately 2 hours.

\subsubsection{Psychophysics procedure}

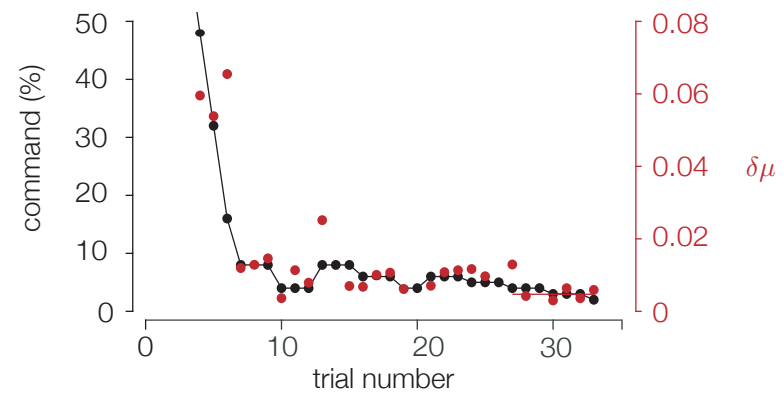

Fig. 5. Typical 3-down/1-up adaptive staircase procedure. The desired friction modulation amplitude is plotted in black and the resulting friction variation amplitude $\delta \mu$ is plotted in red. The red line reports the mean value of the last 6 trials.

Participants sat in a chair in front of the experimental desk and wore headphones with pink noise to prevent any auditory cues from the device. They put their right index finger into the position-tracking apparatus. They were asked to continuously explore the glass plate with their finger by moving back and forth from left to right while synchronizing their movement with a cursor presented on a screen that imposed the finger velocity. After each trial, feedback on the normal force applied to the glass plate was displayed on-screen. They were asked to keep the normal force between 0.4 and $0.8 \mathrm{~N}$. For each trial, they had to perform 2 successive explorations on the glass plate ( 2 backand-forth movements). One of the explorations randomly contained the haptic stimulus (modulated friction) whereas the other one was smooth (constant friction) acting as a reference. Both the stimulus and the reference had the same average friction level. The psychophysical method was a two-alternative forced choice (2AFC): the participants had to report which of the two explorations presented the most irregular texture.

They could answer via an interface on a laptop situated to their left. For each spatial period, the detection threshold, i.e., the minimal perceptible amplitude, was evaluated according to a 3-down/1-up adaptive staircase procedure. After 3 successive correct answers, the amplitude of the stimulus decreased, and after one wrong answer, the amplitude of the stimulus increased. This algorithm converges to the detection threshold. In our experiment, the procedure was stopped after 6 reversals. After every second reversal, the step size was divided by two. To quicken the convergence, the procedure started with a simple 1-down/1-up process until the first reversal. A typical procedure is presented in Fig. 5.

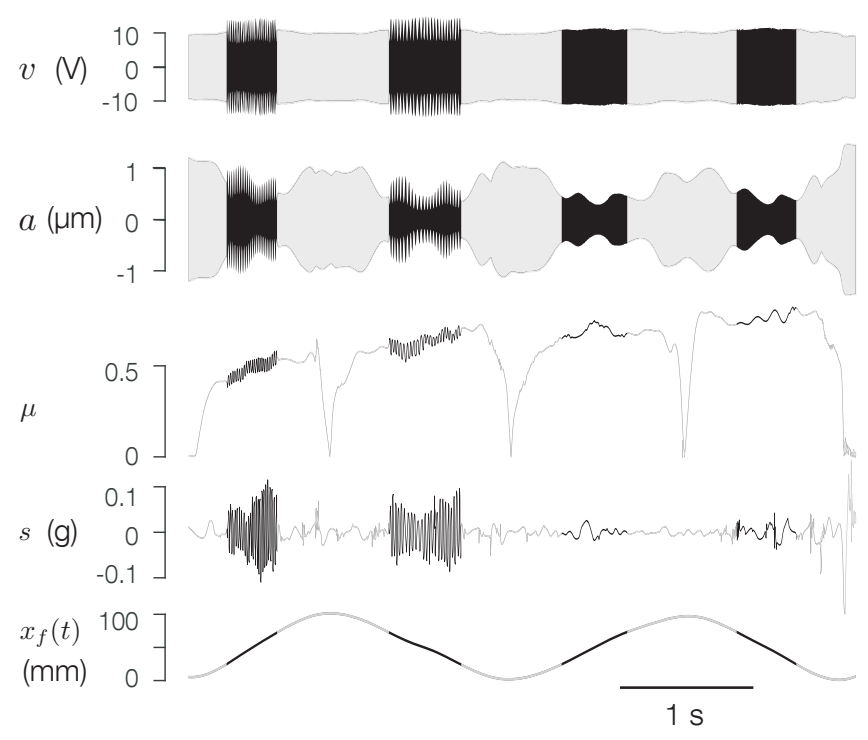

Fig. 6. Raw data measured during one trial. The subject explores the virtual texture with 2 successive lateral back-and-forth movements across the plate. In this trial, the stimulus was presented during the first lateral movement. During the other one, friction reduction was constant. $v$ is the input voltage provided to the piezoelectric actuators, $a$ is the glass plate vibration and $\mu$ is the friction coefficient between the plate and the finger. $s$ is the vibration of the finger, assessed on the first phalanx. $x_{f}$ is the position of the finger. Darkened parts are the selections for which the variations of these variables are calculated

\subsection{Data analysis}

\subsubsection{Signal processing}

The friction coefficient was computed from the absolute value of the tangential force divided by the normal force which was filtered with a $100 \mathrm{~Hz}$ low-pass filter to remove fluctuations. For each trial, the finger position, input voltage to the piezoelectric actuators, glass plate vibrations, friction coefficient and vibrations of the finger were recorded. Fig. 6 presents typical curves of these measurements. Data are first partitioned to isolate the part that corresponds to the $48 \mathrm{~mm}$ length area at the centre of the plate, where the stimulus is (or is not) presented. The finger velocity is assumed to be constant in this part. For each trial, we thus obtain 4 samples: 2 explorations with the haptic stimulus, one in the left-to-right direction and the other in the right-toleft direction, and 2 explorations with a constant actuation which will be used as a references. Calculation methods presented in the next sections are also performed on these references to measure the noise floor. This noise is partly caused by the sensor variations and mainly produced by fluctuations of the frictional force during the exploration of the unactuated surface.

Transfer functions of the block diagram Fig. 2 are calculated by taking the ratio between the mean value of two successive variables for each stimulus frequency. They show how each variable affects the next one as a function the stimulus frequency. 


\subsubsection{Estimation of friction variations}
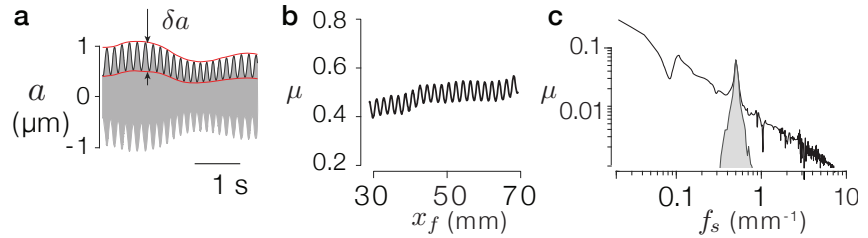

Fig. 7. a. Vibration of the glass plate as a function of time. The envelope of the carrier is in black. The glass plate vibration modulation $\delta a$ is measured by averaging the difference between the upper and lower red envelopes. b. Friction coefficient as a function of the finger position. c. Spatial spectrum of the friction signal. A bandpass filter centred around the spatial frequency of the stimulus is applied to the spatial signal to extract the energy of the signal in the frequency band of interest.

The friction coefficient is interpolated on regularly sampled spatial coordinates system ( $0.01 \mathrm{~mm}$ sampling interval) to obtain the signal as a function of the finger position, as presented in Fig. 7.b. As seen in Fig 7.c, the friction coefficient is entailed with a large $1 / f$ background noise, which is induced by the stochastic interaction between the finger and the plate [17]. Because the friction variations at some thresholds are very close to the noise floor and do not stand out using envelope detection algorithms, we used the spatial frequency domain method illustrated in Fig. 7.c. The spatial signal $\mu_{f}$ is filtered with a bandpass filter around the spatial frequency of the stimulus (2ndorder Butterworth with cut-off frequencies of 0.7 and 1.3 times the stimulus spatial frequency). The energy is then computed with $E_{\mu}=\int\left|\mu_{f}(x)\right|^{2} d x$ of the peak provided by the stimulus. The friction variation amplitude is then assessed by calculating the theoretical amplitude $A$ of a sinewave with the same energy $\delta \mu=\sqrt{2 E_{h} / L}$, with $L=48 \mathrm{~mm}$ being the length of the signal. We selected only the maximal value $\delta \mu$ between the left-to-right direction and right-to-left direction. Indeed, many subjects reported that they sometimes felt the stimulus in only one direction. For each subject and each condition, the smallest perceptible friction variation was defined as the average of $\delta \mu$ over the last 6 trials.

\subsubsection{Skin vibration induced by friction fluctuations}

Vibrations that propagates on the surface of the finger skin, are measured by the an accelerometer and are processed in a way similar to that for the friction coefficient. Partitioned data are bandpass-filtered with the centre frequency corresponding to the resultant frequency of the stimulus according to the velocity condition. The vibration energy in the finger is thus given by $E_{v}=\int\left|a_{f}(t)\right|^{2} d t$.

For the input voltage of the piezoelectric actuator and the glass plate vibration, the envelope of each signal is computed using the Hilbert transform to isolate the modulation from the $35 \mathrm{kHz}$ carrier signal, as shown in Fig. 7.a.

\section{Results}

\subsection{Subject performances}

Explorations were performed with a normal force of a mean value and standard deviation of $0.68 \pm 0.19 \mathrm{~N}$, which is in line with the requested range of 0.4 to $0.8 \mathrm{~N}$. We noticed that subjects tended to increase their normal exploration force as the stimuli became more subtle. The effective velocity of the finger was $55 \pm 10.2 \mathrm{~mm} / \mathrm{s}$ for the low velocity condition and $113.3 \pm 24.3 \mathrm{~mm} / \mathrm{s}$ for the high velocity condition. The spatial periods of the stimuli $(0.125,0.25,0.5,1,2,4$ and $8 \mathrm{~mm}$ ) led to effective frequencies of $440,220,110$, $55,27.5,13.8$ and $6.9 \mathrm{~Hz}$ on average for the low velocity condition and to 906.4, 453.2, 226.6, 113.3, 56.7, 28.3 and $14.2 \mathrm{~Hz}$ on average for the high velocity condition. Each session lasted between 15 and 42 trials (mean 25.4) until the subject converged to their detection threshold.

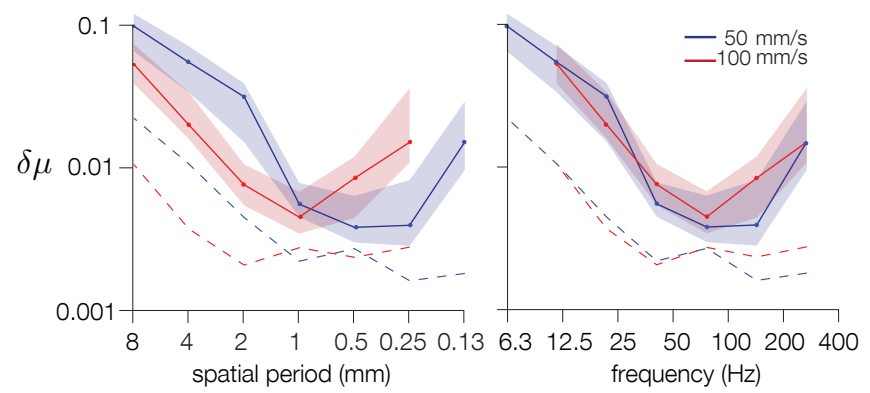

Fig. 8. Friction variation detection thresholds. Curves are presented twice, one in the spatial domain, where the abscissa corresponds to the spatial period of the stimulus (the axis is inverted for comparison), and the other in the frequency domain, where the abscissa corresponds to the frequency given by the ratio between the finger velocity and the spatial period. The solid lines represent the median and the shaded zones represent the first and third quartiles. The dashed lines represent the noise floor calculated on the reference exploration without a stimulus.

\subsection{Tactile thresholds}

For each session, the friction variation $\delta \mu$ is averaged over the last 6 trials of the session to obtain the subjects' friction variation detection threshold for the spatial period. We can thus reconstruct the haptogram -analogous to an audiogram for hearing- of a subject, a curve showing the tactile threshold of the subject at each spatial period as presented in Fig. 8. We decided to show these results in the spatial domain to compare the stimuli and in the frequency domain by taking into account the velocity of the finger.

Statistical analysis was performed on each of these four variables both in the spatial and frequency domains to investigate the effect of the velocity. We performed twoway ANOVAs with the finger velocity and spatial period or frequency as the factors. In the spatial domain, the thresholds are compared according to their spatial period conditions. In the frequency domain, thresholds that share the same frequency during their exploration are compared.

The results showed that the velocity had a significant effect $(\alpha=0.05)$ on the friction variation thresholds in the spatial domain $\left(F_{1,5}=10.43, p=0.0015\right)$ but not in the frequency domain $\left(F_{1,5}=0.13, p=0.72\right)$.

In the same way, plate vibrations $\delta a$ and finger vibrations $\delta s$ were averaged over the last 6 trials of each session to obtain an average value at the subjects' detection thresholds. The results are summarized in Fig. 9. The same trend as for the friction variation threshold curve was observed but with less significance $(\alpha=0.07)$ for the plate vibrations. We observed an effect of the finger velocity on the plate 

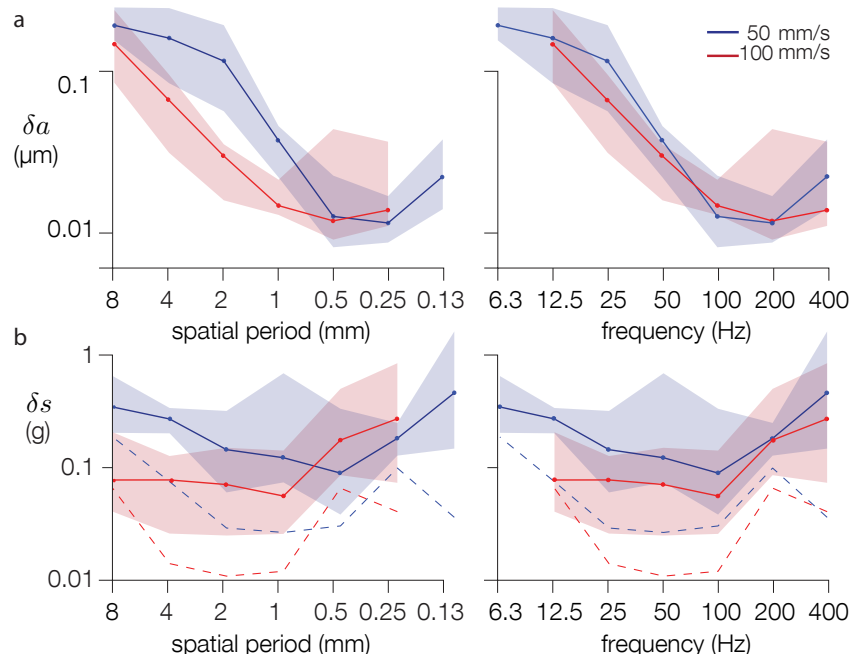

Fig. 9. Values of plate vibration modulation and finger vibration at the detection thresholds in the spatial frequency domains. The solid lines represent the median and the shaded zones represent the first and third quartiles. Upon finger vibration, the dashed lines represent the noise floor calculated on the reference exploration without a stimulus.

vibration modulation at the threshold $\left(F_{1,5}=3.4, p=0.06\right)$ in the spatial domain and no effect $\left(F_{1,5}=0.003, p=0.95\right)$ in the frequency domain. There was no effect of finger velocity on finger vibrations at the thresholds in both the spatial $\left(F_{1,5}=0.45, p=0.5\right)$ and temporal $\left(F_{1,5}=1.88\right.$, $p=0.17)$ domains.

To investigate the relevance of each variable to human perception, we measured their inter-subject variability. We assumed that a variable with less variability among participants would be the most relevant descriptor of tactile perception. We measured for each variable the coefficient of variations $c$ averaged over all velocities and spatial period conditions. We found that the lowest variability was obtained for friction variations $c_{\delta f}=0.42$ whereas the variability for plate vibration modulation was $c_{\delta a}=0.73$ and $c_{\delta s}=0.55$ for finger vibrations. We assume that these data demonstrate that friction variation is the most relevant variable encoding human tactile sensitivity.

\subsection{Transfer functions}

As mentioned in the introduction, the rendering of a haptic stimulus follows several steps, summarized in the block diagram in Fig. 2. Each transfer function is calculated by averaging the data of all the trials, not only at the threshold, as their relationship is assumed to be linearly dependent on the input amplitude. Knowledge on these transfer functions provides a clearer picture of the role of each element involved in the rendering of surface-haptic stimuli.

The transfer function T (Fig. 10.a) shows how the deformations of the piezoelectric actuators make the glass plate vibrate. It reflects the frequency bandwidth of the glass plate vibration modulation. It has been noted before that due to the resonance of the glass plate, the amplitude modulation of the carrier wave will be attenuated for high frequencies. This effect is most noticeable when a step modulation function produces an exponential ring down of the plate oscillations [41]. The attenuation is affected by the plate material and acts as a low-pass filter with a cut-off frequency of $100 \mathrm{~Hz}$ in this particular setup.

The transfer function $\mathbf{F}$ (Fig. 10.b) illustrates how the glass plate vibration affects friction between the finger and the glass plate. $\mathbf{F}$ characterizes the bio-mechanics of the skin-glass contact. The squeeze film effect at the origin of this phenomenon has been explained and modelled [28], but the relationship between the modulation and the friction during an exploration is still subject to questioning. Interestingly, the transfer function $\mathbf{F}$ of this device is not constant. It shows that high and low modulation frequencies of the glass plate vibration are more effective to render strong friction variations.

The last transfer function V (Fig. 10.c) reflects how friction variations propagate into the finger as mechanical vibrations. The curve presents a U-shape with a peak transmissibility of the vibration around $100 \mathrm{~Hz}$, in line with previous studies [42].

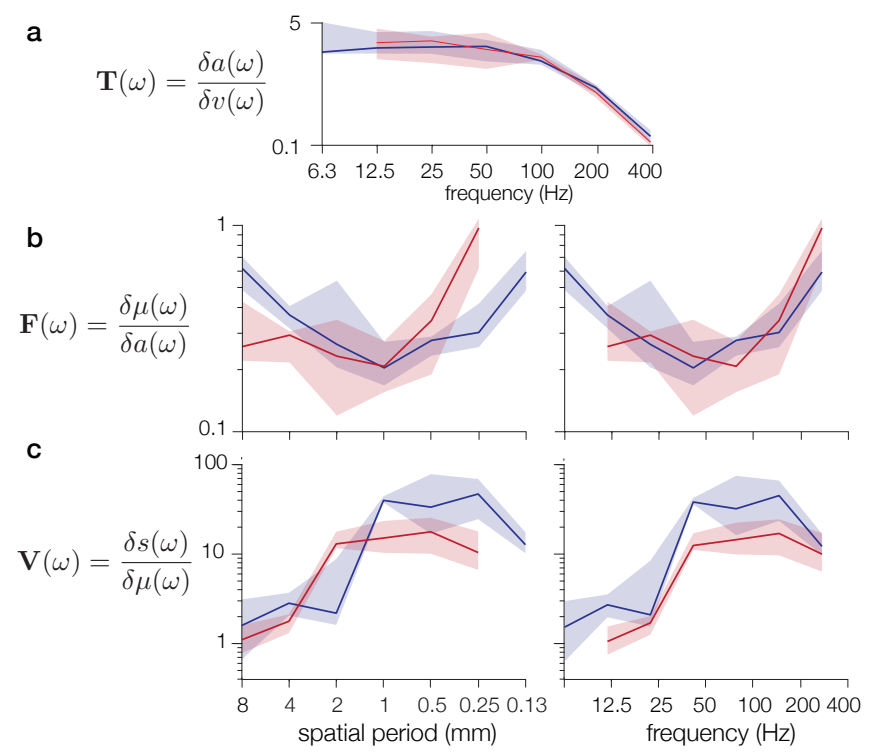

Fig. 10. Transfer function between the different variables from actuation to perception. Data are measured on all trials. The solid lines represent the median and the shaded zone represents the first and third quartiles. a Transfer function between the input voltage modulation of the piezoelectric actuator and the actual vibration variations of the plate. $\mathbf{b}$ Effect of the modulation of the ultrasonic amplitude on the variation of friction, represented in the spatial and frequency domains. c Vibrations measured at the skin level of a given change in frictional force.

\section{Discussion}

Two main results emerge from the experiments presented Fig. 8. The finger velocity influences the detection thresholds of spatially based gratings, but no significant effect appears if we compare these thresholds according to the induced frequency. In addition, threshold distributions across frequencies are not linear and show an optimum sensitivity between 100 and $200 \mathrm{~Hz}$, with a decrease in sensitivity for higher frequencies. This observation suggests that friction-modulated textures are temporally integrated, since the detection threshold is not affected by the scanning speed, meaning that the frequency model (Fig. 1.b) is preferred over the spatial model hypothesis (Fig. 1.a). 
Friction-modulated grating detection thresholds follow similar trends to those of vibrotactile detection, and therefore, the vast literature on vibrotactile perception can support the design of meaningful friction-modulated stimuli. Pacinians are probably the mechanoreceptors that are the most involved in the detection of friction-modulated textures since their optimal sensitivity $(250 \mathrm{~Hz})$ is quite similar to the detection threshold curves (Fig. 8).

An interesting consequence of this result is that it exists an optimal combination of the spatial period and scanning velocity that enhances the tactile sensation of a frictionmodulated texture. Small-scale synthetic textures are better perceived with low-velocity exploration whereas large relief perception is improved by faster explorations. A frictionmodulated grating with a given spatial period can have the same detection threshold as that of another grating with a spatial period that is half the size if explored twice as fast.

It stands out that the stimuli used in the experiment may not be significantly different, from a perceptual point of view, from spatially encoded vibrotactile feedback with the same envelope. One major limitation of this result is that, as we investigated detection thresholds, the haptic effects were very subtle. This could be the reason why they are perceived more like vibrations than reliefs. This phenomenon might not be the same for gratings with higher amplitude.

The detection thresholds could have been altered by fatigue. It has been shown that tactile sensitivity is affected by the stimulation duration [43]. We mitigated this issue by asking subjects to remove their finger from the glass plate between each trial, and imposing breaks.

Since the inter-subject variability is the lowest for the friction-variation variable, this variable seems to be the most relevant for controlling perception, in line with previous studies [44]. For example, some subjects needed higher plate vibration amplitudes at their detection threshold than other subjects, while the friction variation thresholds were similar between subjects. This is reflected in the relatively large variability of the transfer function $F$ (Fig. 10.b) when compared to the vibratory transfer function $V$. The variability between plate vibration and friction reduction efficiency reflects differences in the bio-mechanical properties of the finger skin, which were also observed in [45] and have been shown to significantly affect subjects' perception [46]. Therefore, stimuli generation on surface haptics should focus on controlling the frictional force and friction coefficient rather than the ultrasonic amplitudes, as proposed in [47], [48].

Furthermore, the ratio between the perceptual thresholds and the amplitude of the noise floor while sliding (acquired when no stimuli are presented) is relatively constant, at least for low frequencies. Thus, the frictional noise due to contact could be the limiting factor in the estimation of friction-modulated textures.

The finger vibrations at the detection thresholds presented in Fig. 9 show considerable variability. This is probably because the measured signals are close to the noise floor. Vibrations are attenuated by the skin during their propagation from the contact area to the sensor. The transfer function $\mathbf{V}(\omega)$ between the friction variations and the finger vibration (Fig. 10.c) is more reliable because it is measured for all trials and not only with low amplitudes at the threshold. Vibrations are maximally produced by friction changes between 100 and $200 \mathrm{~Hz}$, which could explain the increased sensitivity in this frequency range.

The transfer function $\mathbf{F}(\omega)$, shown in Fig. 10.b, reveals that a given amplitude of ultrasonic vibration modulates the frictional force more effectively at high frequencies. This boost in high frequencies naturally and conveniently compensates the attenuation of the amplitude of the plate vibration at high modulation frequencies. The attenuation of the modulation of the ultrasonic vibration amplitude is due to the resonant behavior of the glass plate [41] and is clearly illustrated in Fig. 10.a. The combination of both effects might explain why it is possible to render perceptible friction modulation feedback at frequencies above the cutoff frequency of the glass plate.

\section{Conclusions}

The objective of the present study was to investigate the detection thresholds of spatially defined fiction-modulated textures and to examine whether these textures were perceived more like real relief or like vibrations. We investigated the detection thresholds of spatial friction-modulated sinusoidal gratings at 7 spatial periods and for two exploratory velocity conditions because the scanning speed determines the frequency of the generated vibrations.

We found that the velocity had no effect on the thresholds if we compare the stimuli with their resulting vibration frequencies given by the ratio between the velocity and the spatial signal period. The detection threshold curves resulted in a U-curve with an optimal sensitivity between 100 and $200 \mathrm{~Hz}$. These results demonstrate similar detection thresholds between ultrasonic friction-modulated gratings and vibrotactile ones.

Friction variations were measured with a one-of-a-kind force sensor using interferometry to provide unmatched precision over a frequency range spanning continuous forces up to kilohertz force fluctuations. Analyses of the transfer function between the different relevant variables provided some clues that could explain the curve shape for the thresholds. This study improves our understanding of the impact of the exploration speed on the perception of virtual features on haptic touchscreens.

\section{ACKNOWLEDGMENTS}

The authors would like to thank Sebastien Denjean and Vincent Roussarie for their thoughtful comments on the experimental design, Julien Diperi and Nicolas Huloux for their support in the setup design, and Etienne Thoret for his advice on the data analysis.

\section{REFERENCES}

[1] S. Okamoto, M. Konyo, S. Saga, and S. Tadokoro, "Detectability and perceptual consequences of delayed feedback in a vibrotactile texture display," IEEE Transactions on Haptics, vol. 2, no. 2, pp. 7384, 2009.

[2] M. Wiertlewski, J. Lozada, and V. Hayward, "The spatial spectrum of tangential skin displacement can encode tactual texture," IEEE Transactions on Robotics, vol. 27, no. 3, pp. 461-472, 2011.

[3] S. J. Lederman, J. M. Loomis, and D. A. Williams, "The role of vibration in the tactual perception of roughness," Perception $\mathcal{E}$ Psychophysics, vol. 32, no. 2, pp. 109-116, 1982. 
[4] S. J. Bensmaia and M. Hollins, "The vibrations of texture," Somatosensory \& motor research, vol. 20, no. 1, pp. 33-43, 2003.

[5] S. Bochereau, S. Sinclair, and V. Hayward, "Perceptual constancy in the reproduction of virtual tactile textures with surface displays," ACM Transactions on Applied Perception (TAP), vol. 15, no. 2, p. 10, 2018.

[6] R. T. Verrillo, A. J. Fraioli, and R. L. Smith, "Sensation magnitude of vibrotactile stimuli," Perception \& Psychophysics, vol. 6, no. 6, pp. 366-372, 1969.

[7] R. T. Verrillo, "Psychophysics of vibrotactile stimulation," The Journal of the Acoustical Society of America, vol. 77, no. 1, pp. 225$232,1985$.

[8] S. Louw, A. M. Kappers, and J. J. Koenderink, “Haptic detection thresholds of gaussian profiles over the whole range of spatial scales," Experimental brain research, vol. 132, no. 3, pp. 369-374, 2000.

[9] H. T. Nefs, A. M. Kappers, and J. J. Koenderink, “Amplitude and spatial-period discrimination in sinusoidal gratings by dynamic touch," Perception, vol. 30, no. 10, pp. 1263-1274, 2001.

[10] S. J. Bolanowski Jr, G. A. Gescheider, R. T. Verrillo, and C. M. Checkosky, "Four channels mediate the mechanical aspects of touch," The Journal of the Acoustical society of America, vol. 84, no. 5, pp. 1680-1694, 1988.

[11] A. I. Weber, H. P. Saal, J. D. Lieber, J.-W. Cheng, L. R. Manfredi, J. F. Dammann, and S. J. Bensmaia, "Spatial and temporal codes mediate the tactile perception of natural textures," Proceedings of the National Academy of Sciences, vol. 110, no. 42, pp. 17107-17112, 2013.

[12] D. Katz, The world of touch. Psychology press, 2013.

[13] C. J. Cascio and K. Sathian, "Temporal cues contribute to tactile perception of roughness," Journal of Neuroscience, vol. 21, no. 14, pp. 5289-5296, 2001.

[14] M. Hollins and S. R. Risner, "Evidence for the duplex theory of tactile texture perception," Perception \& psychophysics, vol. 62, no. 4, pp. 695-705, 2000.

[15] B. Unger, R. Hollis, and R. Klatzky, "Roughness perception in virtual textures," IEEE Transactions on Haptics, vol. 4, no. 2, pp. 122-133, 2010.

[16] S. J. Lederman, "Tactile roughness of grooved surfaces: The touching process and effects of macro-and microsurface structure," Perception \& Psychophysics, vol. 16, no. 2, pp. 385-395, 1974.

[17] M. Wiertlewski, C. Hudin, and V. Hayward, "On the $1 / \mathrm{f}$ noise and non-integer harmonic decay of the interaction of a finger sliding on flat and sinusoidal surfaces," in 2011 IEEE World Haptics Conference. IEEE, 2011, pp. 25-30.

[18] R. L. Klatzky and S. J. Lederman, "Perceiving texture through a probe," Touch in virtual environments, pp. 180-193, 2002.

[19] D. J. Meyer, M. A. Peshkin, and J. E. Colgate, "Tactile paintbrush: A procedural method for generating spatial haptic texture," in 2016 IEEE Haptics Symposium (HAPTICS). IEEE, 2016, pp. 259-264.

[20] H. Culbertson and K. J. Kuchenbecker, "Should haptic texture vibrations respond to user force and speed?" in 2015 IEEE World Haptics Conference (WHC). IEEE, 2015, pp. 106-112.

[21] A. Dépeault, E.-M. Meftah, and C. E. Chapman, "Tactile speed scaling: contributions of time and space," Journal of neurophysiology, vol. 99, no. 3, pp. 1422-1434, 2008.

[22] B. P. Delhaye, M. K. O'Donnell, J. D. Lieber, K. R. McLellan, and S. J. Bensmaia, "Feeling fooled: Texture contaminates the neural code for tactile speed," PLoS biology, vol. 17, no. 8, 2019.

[23] I. Poupyrev and S. Maruyama, "Tactile interfaces for small touch screens," in Proceedings of the 16th annual ACM symposium on User interface software and technology. ACM, 2003, pp. 217-220.

[24] Y. Visell, J. R. Cooperstock, B. L. Giordano, K. Franinovic, A. Law, S. McAdams, K. Jathal, and F. Fontana, "A vibrotactile device for display of virtual ground materials in walking," in International Conference on Human Haptic Sensing and Touch Enabled Computer Applications. Springer, 2008, pp. 420-426.

[25] H.-Y. Chen, J. Park, S. Dai, and H. Z. Tan, "Design and evaluation of identifiable key-click signals for mobile devices," IEEE Transactions on Haptics, vol. 4, no. 4, pp. 229-241, 2011.

[26] L. Panter and C. Hudin, "Sparse actuator array combined with inverse filter for multitouch vibrotactile stimulation," in 2019 IEEE World Haptics Conference (WHC). IEEE, 2019, pp. 19-24.

[27] C. Shultz, M. Peshkin, and J. E. Colgate, "The application of tactile, audible, and ultrasonic forces to human fingertips using broadband electroadhesion," IEEE transactions on haptics, vol. 11, no. 2, pp. 279-290, 2018.
[28] M. Wiertlewski, R. F. Friesen, and J. E. Colgate, "Partial squeeze film levitation modulates fingertip friction," Proceedings of the national academy of sciences, vol. 113, no. 33, pp. 9210-9215, 2016.

[29] L. Winfield, J. Glassmire, J. E. Colgate, and M. Peshkin, "T-pad: Tactile pattern display through variable friction reduction," in Second Joint EuroHaptics Conference and Symposium on Haptic Interfaces for Virtual Environment and Teleoperator Systems (WHC'07). IEEE, 2007, pp. 421-426.

[30] M. Biet, F. Giraud, and B. Lemaire-Semail, "Squeeze film effect for the design of an ultrasonic tactile plate," IEEE transactions on ultrasonics, ferroelectrics, and frequency control, vol. 54, no. 12, pp. 2678-2688, 2007.

[31] D. Gueorguiev, E. Vezzoli, T. Sednaoui, L. Grisoni, and B. LemaireSemail, "The perception of ultrasonic square reductions of friction with variable sharpness and duration," IEEE transactions on haptics, 2019.

[32] M. K. Saleem, C. Yilmaz, and C. Basdogan, "Psychophysical evaluation of change in friction on an ultrasonically-actuated touchscreen," IEEE transactions on haptics, 2019.

[33] J. Monnoyer, E. Diaz, C. Bourdin, and M. Wiertlewski, "Perception of ultrasonic switches involves large discontinuity of the mechanical impedance," IEEE transactions on haptics, vol. 11, no. 4, pp. 579-589, 2018.

[34] C. Bernard, J. Monnoyer, and M. Wiertlewski, "Harmonious textures: the perceptual dimensions of synthetic sinusoidal gratings," in International Conference on Human Haptic Sensing and Touch Enabled Computer Applications. Springer, 2018, pp. 685-695.

[35] R. F. Friesen, R. L. Klatzky, M. A. Peshkin, and J. E. Colgate, "Single pitch perception of multi-frequency textures," in 2018 IEEE Haptics Symposium (HAPTICS). IEEE, 2018, pp. 290-295.

[36] A. Isleyen, Y. Vardar, and C. Basdogan, "Tactile roughness perception of virtual gratings by electrovibration," IEEE Transactions on Haptics, 2019.

[37] W. B. Messaoud, M.-A. Bueno, and B. Lemaire-Semail, "Relation between human perceived friction and finger friction characteristics," Tribology International, vol. 98, pp. 261-269, 2016.

[38] O. Bau, I. Poupyrev, A. Israr, and C. Harrison, "Teslatouch: electrovibration for touch surfaces," in Proceedings of the 23nd annual ACM symposium on User interface software and technology. ACM, 2010, pp. 283-292.

[39] Y. Vardar, B. Güçlü, and C. Basdogan, "Effect of waveform on tactile perception by electrovibration displayed on touch screens," IEEE transactions on haptics, vol. 10, no. 4, pp. 488-499, 2017.

[40] Y. Shao, V. Hayward, and Y. Visell, "Spatial patterns of cutaneous vibration during whole-hand haptic interactions," Proceedings of the National Academy of Sciences, vol. 113, no. 15, pp. 4188-4193, 2016.

[41] D. J. Meyer, M. Wiertlewski, M. A. Peshkin, and J. E. Colgate, "Dynamics of ultrasonic and electrostatic friction modulation for rendering texture on haptic surfaces," in 2014 IEEE Haptics Symposium (HAPTICS). IEEE, 2014, pp. 63-67.

[42] Y. Tanaka, Y. Horita, and A. Sano, "Finger-mounted skin vibration sensor for active touch," in International Conference on Human Haptic Sensing and Touch Enabled Computer Applications. Springer, 2012, pp. 169-174.

[43] J. Hahn, "Vibrotactile adaptation and recovery measured by two methods." Journal of Experimental Psychology, vol. 71, no. 5, p. 655, 1966.

[44] A. M. Smith, C. E. Chapman, M. Deslandes, J.-S. Langlais, and M.$P$. Thibodeau, "Role of friction and tangential force variation in the subjective scaling of tactile roughness," Experimental brain research, vol. 144, no. 2, pp. 211-223, 2002.

[45] A. Kaci, A. Torres, F. Giraud, C. Giraud-Audine, M. Amberg, and B. Lemaire-Semail, "Fundamental acoustical finger force calculation for out-of-plane ultrasonic vibration and its correlation with friction reduction," in 2019 IEEE World Haptics Conference (WHC). IEEE, 2019, pp. 413-418.

[46] J. Monnoyer, E. Diaz, C. Bourdin, and M. Wiertlewski, "Optimal skin impedance promotes perception of ultrasonic switches," in 2017 IEEE World Haptics Conference (WHC). IEEE, 2017, pp. 130135.

[47] N. Huloux, J. Monnoyer, M. Boyron, and M. Wiertlewski, “Overcoming the variability of fingertip friction with surface-haptic force-feedback," in International Conference on Human Haptic Sensing and Touch Enabled Computer Applications. Springer, 2018, pp. 326-337. 
[48] W. B. Messaoud, M. Amberg, B. Lemaire-Semail, F. Giraud, and M.-A. Bueno, "High fidelity closed loop controlled friction in smarttac tactile stimulator," in 2015 17th European Conference on Power Electronics and Applications (EPE'15 ECCE-Europe). IEEE, 2015, pp. 1-9.

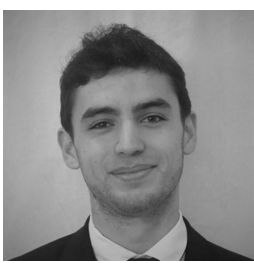

Corentin Bernard graduated from the Ecole Centrale de Marseille in 2017 and holds a Masters degree in acoustics from the Aix-Marseille Université. Since 2018, he is a doctoral student in the Groupe PSA and two laboratories at the University of Aix-Marseille: the Perception, Representations, Image, Sound and Music Laboratory (PRISM) and the Institute of Movement Sciences (ISM). He is interested in understanding how humans perceive haptic feedback and the sounds associated with it. His work focuses on psychophysics to better understand human perception of haptic surfaces, audio-haptic multimodal integration, synthesis of haptic signal and human-machine interfaces. His work has been nominated for Best paper at the 2018 Eurohaptics conference in Pisa, Italy.

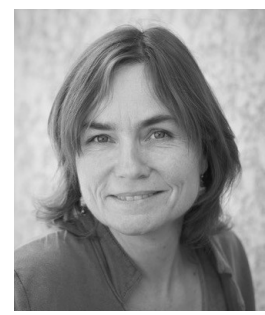

Sølvi Ystad received her degree as a civil engineer in electronics from NTH (Norges Tekniske Høgskole), Norway in 1992. In 1998 she received her Ph.D. degree in acoustics from the University of Aix-Marseille II, Marseille. After a post doctoral stay at the University of Stanford CCRMA, California, she obtained a researcher position at the CNRS (Centre National de la Recherche Scientifique) in Marseille in 2002. In 2017 she co-founded the interdisciplinary artscience laboratory PRISM - Perception, Representations, Image, Sound, Music (www.prism.cnrs.fr) in Marseille. Her research activities mainly focus on investigations of auditory perception through so-called perceptual engineering which consists of crossing different disciplines to link physical and signal knowledge with human perception and cognition.

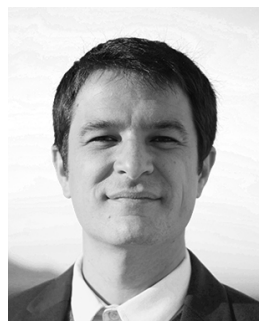

Michaël Wiertlewski is Assistant Professor at the Cognitive Robotics Department of the TU Delft in the Netherlands. He obtained his Ph.D. degree at the Universite Pierre et Marie Curie under the auspices of the Laboratoire d'Intégration des Systèmes et des Technologies of the Commissariat l'Energie Atomique (CEALIST) in Fontenay-aux-Roses, France. In 2012 he joined the Neuroscience and Robotics $(\mathrm{NxR})$ lab at Northwestern University in Evanston, IL, USA where he studied the physics of ultrasonic friction-modulation. From 2015 to 2019 he was CNRS Chargé de Recherche at Aix-Marseille university. He received the Early Career Award from the Technical Committee on Haptics in 2017. His main research interests include the design of tactile interfaces, the physics of the skin/surface interaction and tactile perception.

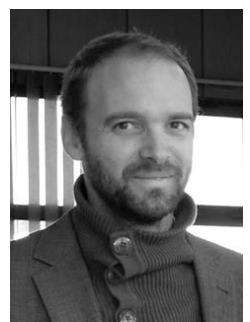

Jocelyn Monnoyer is a research engineer at the Department for Human Perception of the Groupe PSA since 2005. He was involved in various aspects of designing a car including metal stamping and the exterior design and spent several years as a member the virtual reality department of PSA Group. He now coordinates the OpenLab joint research center between Aix-Marseille University and PSA Group. He is responsible for fostering public and private research partnerships around the topics related to human factors. In 2016, he started his PhD studies in haptic perception at Aix-Marseille University. His work focuses on ultrasonic virtual switches and he was awarded the Best Student Paper at the 2016 Eurohaptics Conference in London and the 2018 IEEE Transactions on Haptics Best Paper Award. 